\title{
Technikfolgenabschätzung als technikwissenschaftliche Disziplin?
}

Methodenmix und Modellbildung

Klaus Kornwachs, Büro für Kultur und Technik, Strickers Höhe 22, 88260 Argenbühl (klaus@kornwachs.de)

Aufgrund ihrer Methode wie auch Forschungspraxis kann die Technikfolgenabschätzung (TA) als eine Teildisziplin der Technikwissenschaften angesehen werden kann. Dies zeigt sich vor allem dann, wenn man den methodischen Kern der Technikfolgenabschätzung mit den Ergebnissen der wissenschaftstheoretischen Analyse der technikwissenschaftlichen Disziplinen vergleicht. Das Problem des Methodenmix und der Modellbildung stellt sich in gleicher Weise wie in den Technikwissenschaften und kann hier wie da auch wissenschaftlich basiert gelöst werden.

\section{Technology assessment as a discipline of technosciences}

Mix of methods and modeling

With respect to its methods and research practice, technology assessment (TA) may be regarded as a sub-discipline of the technosciences. This becomes evident when comparing the methodological core of TA with the results of the epistemological analysis of technoscientific disciplines. The problem of mixing methods and modeling has the same structure within the technosciences and can be solved with the same scientific methods.

Keywords: technology assessment, methodology, philosophy of applied science, technosciences

This is an article distributed under the terms of the Creative Commons Attribution License CCBY 4.0 (https://creativecommons.org/licenses/by/4.0/)

https://doi.org/10.14512/tatup.27.1.46

Submitted: 17.10.2017. Peer reviewed. Accepted: 26.01.2018
In memoriam Günter Ropohl (1939-2017)

\section{Einleitung}

Die These dieses Beitrags ist, dass die Technikfolgenabschätzung (TA) hinsichtlich ihrer Methodik, ihres Umfangs und ihres Gegenstands eine Disziplin der Technikwissenschaft sein kann, da sie rational-systematische Vorgehensweisen verwendet, wie sie in den Gestaltung- und Analyseverfahren der Technikwissenschaften vorkommen. Da TA zwangsläufig interdisziplinär ist, muss sie die jeweiligen wissenschaftlichen Standards der beteiligten Disziplinen berücksichtigen. Trotz ihres tentativen Grundzugs muss sie einen theoretischen Kern haben, der die Verwendung ihrer Methoden und Verfahren rechtfertigt. TA ist darüber hinaus als Praxis auch an die Bedingungen und Möglichkeiten der Beratung für Politik, Gesellschaft und Wirtschaft gebunden und damit gelten auch für sie die zum Teil schon erarbeiteten, zum Teil noch weiter zu entwickelnden ethischen Standards für die Technikwissenschaften sowie des Ingenieurs- und Beratungswesen.

\section{Theoretische Versuche}

Es gibt in der Bemühung, TA theoretisch zu verstehen, drei Grundrichtungen:

In der ersten Grundrichtung ist TA als eine wissenschaftliche Beratungspraxis zu sehen (Decker 2007, S. 31). TA ist in diesem Verständnis ,eine spezifische Transferleistung des Wissenschaftssystems an außerwissenschaftliche Adressaten" (Grunwald 2007 a, S. 14). Die Theorie der TA bezieht sich dann auf eine wissenschaftstheoretische Untersuchung der Tragweite der Begriffe „Beratung“, „Wissenschaftlichkeit“, „Wissenschaftskommunikation und -Interaktion“, „Folgen“ etc. Hier sind nun zwei Untervarianten denkbar: 
a) Durch eine gewisse Schulenbildung dominiert das wissenschaftstheoretische Verständnis des methodischen Rekonstruktivismus, das die Begründung von Wissenschaft aus der lebensweltlichen Praxis, der praktischen Bewährung und aus dem methodischen Reden über das Handeln aufbaut (Janich 1997).

b) Es ist ebenso möglich, TA als eine gesellschaftliche Praxis zu verstehen, die im Rahmen soziologischer Theorie- und Modellbildungen gesehen werden kann. Diese wiederum weisen ein sehr ausdifferenziertes Spektrum auf, angefangen von der Luhmannschen Theorie der Sozialen Systeme über Agenten-Modelle, Design Thinking und Grounded Theory hin zur Adaption der wirtschaftswissenschaftlichen Ansätze der Verhaltensökonomie. Hinzu gesellt sich die Diversität der wissenschaftstheoretischen Auffassungen in den gesellschaftswissenschaftlichen Disziplinen.

Als zweite Grundrichtung kann man wissenschaftstheoretisch TA als ein Forschen über Technik ansehen, wenn man Technik, wie dies Ropohl (1999) tut, nicht nur aus Artefakten bestehend begreift, sondern die Konzeption, Entstehung, Herstellung und Verwendung bis hin zur Entsorgung von Artefakten betrachtet und auch die organisatorischen wie institutionellen Rahmenbedingungen als mit zur Technik zugehörend ansieht. TA ist dann die Untersuchung der Technisierung (Ropohl 2007, S. 115) und bedarf daher einer Theorie. Grundlage hierfür ist nach Ropohl eine Systemtheorie, die eine geeignete Beschreibung der Technisierung erlaubt.

Drittens ist TA als problemorientierte Forschung anzusehen (Grunwald 2007 b, S.6), deren Methoden und Ergebnisse im Kontext ihrer Beauftragung und Adressaten gesehen werden muss. Bereits zu Zeiten des US-amerikanischen Office of Technology Assessment (OTA) sind solche Ansätze diskutiert worden, insbesondere dass TA angewandte Forschung sei (Shrader-Frechette 1980), wenngleich es damals noch keine weiter ausgebauten Ansätze zu einer Theorie der angewandten Wissenschaften gab.

Man kann in dieser Dreiteilung auch sehen, dass es drei Ebenen der Reflexion über TA gibt, die auch heute noch nicht konsistent oder übergreifend in eine systematisierende Theorie der TA gebracht worden wären. Dies sind nach Grunwald (2007a, S. 11) die Makroebene der gesellschaftstheoretischen Verortung, die Mesoebene der Prämissen, Diagnosen und Zielsetzungen, und die Mikroebene, in der sowohl die TA-Beratungspraxis angesiedelt ist (Grunwald 2007 a, S. 11), aber wohl auch die wissenschaftstheoretischen Fragen nach der Wissenschaftlichkeit und Geeignetheit der gewählten Methoden zu finden sind.

Sucht man einen gemeinsamen Nenner der oben genannten Grundrichtungen, wie TA zu sehen sei, kann man TA als eine mit wissenschaftlichen Mitteln betriebene systematische und nachvollziehbare Gewinnung von Erkenntnissen über Auswirkungen, Folgen und Möglichkeiten der Herstellung, der Verwendung und der Entsorgung bestehender und/oder sich entwickelnder Technologien begreifen. Diese Erkenntnisse können zur Bewertung vorhandener wie zur Gestaltung künftiger Technologien angewendet werden und können in den Technikwissenschaften Anwendung finden. Dies schließt im übrigen Beratungsleistungen zur Technologiepolitik und Bereitstellen des notwendigen Wissens zur Gestaltungspartizipation mit ein (Gethmann und Grunwald 1996). Ist damit TA aber schon eine technikwissenschaftliche Disziplin?

\section{Technikwissenschaften}

Wie die Deutsche Akademie der Technikwissenschaften (acatech) erklärt, schaffen Technikwissenschaften „kognitive Voraussetzungen für Innovation in der Technik und Anwendung technischen Wissens und legen die Grundlagen für die Reflexion ihrer Implikationen und Folgen“ (acatech 2013, S. 8). Die Selbstvergewisserung der Technikwissenschaften, im Besonderen ihre wissenschaftstheoretische Durchdringung, steht jedoch wissenschaftsgeschichtlich wohl erst am Anfang. ${ }^{1}$ Es hat sich die Erkenntnis durchgesetzt, dass Technik eben nicht die bloße Anwendung naturwissenschaftlicher Gesetzlichkeiten darstellt, sondern über weitgehend eigenständige Methoden und Verfahren verfügt. Sie benutzt einerseits die Naturwissenschaften, um zu technologischem Wissen zu gelangen, ist andererseits aber auch Voraussetzung, um Naturwissenschaft überhaupt experimentell betreiben zu können (Grunwald 2007 c). Diese Verfahren finden sich auch in den empirischen Wissenschaften wieder, soweit sie Technologien bei Beobachtungen, Experimenten und Tests benutzen. Allerdings müssen die Technikwissenschaften in besonderer Weise heterogene Wissensbestände unterschiedlicher Herkunft in Modelle integrieren können, um der Komplexität ihres Untersuchungsgegenstands gerecht zu werden. So bestehen die Technikwissenschaften aus vielen Disziplinen, dazu gehören auch die praktischen Bereiche wie Gestaltung, technische Erfahrung und Kreativität, die in gewisser Weise das Material darstellen, aus dem durch Abstraktion Regularitäten und Prinzipien gewonnen werden.

Man kann den Kern der Technikwissenschaften vereinfacht so umschreiben: Technisches Wissen manifestiert sich nach Bunge (1967) in Regeln, formal „Effekt B per Maßnahme A“, wobei das Gewinnen und Begründung dieses Regelwissens sowohl aus Erfahrung als auch aus theoretischen Überlegungen heraus (z. B. weil B aus A folgt) erfolgen kann.

\section{Der Gegenstandsbereich}

Nach dieser Definition kann man Technikfolgenabschätzung formal als die Frage danach ansehen, wie bei einer gegebenen technologischen Regel B per A die „Nebenwirkungen“ B' ausfallen

1 Anfänge bei Bunge (1967, Kap. 11), handlungstheoretische Ansätze bei von Wright (1994), gefolgt von wissenschaftstheoretisch inspirierten Ansätzen (Tetens 1982; Banse et al. 2006, Poser 2017), pragmatischen (Kroes und Meijers 2000) und formalen Versuchen (Kornwachs 2012). 
könnten. Es scheint, als ob Prognosen für eine Technikfolgenabschätzung ,erkenntnistheoretisch geradezu ihr Rückhalt“ (Grunwald 1999, S. 98) darstellen würden. Das methodische Problem liegt zum einen jedoch darin, dass nicht jede Erklärung (z. B. durch eine gesetzesartige Aussage) eine Prognose erlaubt - die Evolutionstheorie erlaubt beispielsweise die Erklärung der Entstehung der Arten, sie kann aber künftige Entwicklungen nicht vorhersagen. Zum anderen ist Prognostizierbarkeit keine hinreichende Voraussetzung für eine Erklärung - jede quantitative Trendextrapolation ist kurzfristig auch ohne theoretisches Verständnis der inneren Dynamik eines Prozesses durch eine signaltheoretische oder statistische Analyse extrapolierbar. Hingegen sind qualitativ definierte Variablen, wie sie z. B. auch in Bewertungs- und Normensystemen vorkommen, die man zur Technikbewertung heranzieht, durch quantitative Methoden weder darstellbar noch prognostizierbar.

Weiterhin muss man die Prognosen des möglichen zukünftigen Verlaufs der Größen, die durch menschliches Handeln beeinflussbar sind, von denen unterscheiden, die dies offenkundig nicht sind. So gibt es den wohlbekannten Effekt, dass die Veröffentlichung einer Prognose einer möglichen Dynamik eben diese selbst ändert, indem sie kontrovers diskutiert wird und beispielsweise Abwehrmaßnahmen ergriffen werden. Man sieht daraus, dass Prognosen in der Technikfolgenabschätzung eher unsere Erwartungshaltungen kennzeichnen.

Deshalb hat man sich seit einiger Zeit angewöhnt, nicht mehr von Zukunft, sondern von ,Zukünften“ zu sprechen (Grunwald 1999; acatech 2012) - man diskutiert über mögliche Szenarien und verzichtet darauf, anzugeben, mit welcher Wahrscheinlichkeit sie eintreten könnten. Dennoch kommen wir wohl nicht darum herum, uns Vorstellungen von Folgen und intendierten wie nichtintendierten Nebenwirkungen von bestehenden oder geplanten Techniken zu machen - ganz egal, wie begrenzt das dazu vorhandene Instrumentarium auch sein mag. Daher ist TA weitaus tentativer angelegt als andere technikwissenschaftliche Disziplinen.

\section{Methoden}

Im Laufe der Praxis von TA-Projekten, beginnend mit den Erfahrungen des OTA, hat sich eine variantenreiche Methodik der Arbeitsschritte herausgebildet, die man wissenschaftstheoretisch jedoch auf einen gewissen methodischen Kern abbilden kann, der sich an den Möglichkeiten der systemtheoretischen Beschreibung orientiert, wie sie im Laufe der 1970er- und 1980erJahre entstanden sind. Man findet diesen Kern systemtheoretischer Beschreibung (Mesarovic 1972) z. B. auch in der im Ingenieursbereich entwickelten Nutzwertanalyse, der Wertanalyse wie bei Konstruktionssystematiken und vielen anderen. Eingebettet in die üblichen Vorgehensweisen des Projektmanagements wie auch der Beratungspraxis findet sich der angesprochene methodische Kern auch bei der Modellbildung in der TA und in den Bewertungsroutinen der Technikbewertung wieder.
Zunächst kann man feststellen, dass TA zu den rational systematischen Methoden gezählt werden kann und sich im Gegensatz zur früheren Einschätzung als einer lediglich heuristischen Methode (Paschen et al. 1978) zu einer Vorgehensweise gewandelt hat, die unter vergleichbaren Bedingungen mit vergleichbaren oder ähnlichen Ergebnissen wiederholt werden kann

\section{TA ist eine rational systematische Methode: eine Vorgehensweise, die unter vergleichbaren Bedin- gungen mit ähnlichen Ergebnissen wiederholt werden kann.}

(Kornwachs 2006, S. 149). Dabei legen meistens die beteiligten Disziplinen die Angemessenheit der gewählten und nachvollziehbaren Verfahren mit dem Gegenstandsbereich fest. Damit stellen die Methoden der TA immer einen Methodenmix dar. Bei rational-systematischen Methoden ist es selbstverständlich, dass die Kriterien für die richtige Durchführung der einzelnen Schritte vorher festgelegt sein müssen und von der konkreten unterschiedlichen Auswahl der einzelnen Fragestellungen und der Durchführungen der Verfahren selbst unabhängig bleiben. Die Grenzen der angewandten Verfahren (Kornwachs 2006, S. 147) müssen durch die Kenntnis der Verfahren und ihrer Begründung, also auch durch die Methodik der TA selbst, bestimmt werden können.

\section{Das Problem beim Methodenmix}

Das Grundproblem bei einem Methodenmix, wie er in TA-Projekten regelmäßig verwendet wird, lässt sich mit drei konfliktbeladenen Bereichen charakterisieren, die Ausgangspunkt von Kontroversen über die richtige Vorgehensweise sein können:

1. Man hat es bei der TA mit unterschiedlichen Teilsystemen und damit Beschreibungsarten zu tun. So ist bei den Gegenstandsbereichen zu unterscheiden zwischen

- einer Technologie (z. B. Big Data) und ihrer künftigen Entwicklung (z. B. Konvergenz mit KI-Technologien),

- der Organisationsweise ihrer Anwendung (z. B. bei der Analyse von Sozialverhalten in Städten),

- dem absehbaren Gebrauch (einschließlich dual use und Missbrauchsmöglichkeiten, z. B. zur Steuerung von Smart Cities oder zur Erkennung unliebsamer politischer Aktivitäten) und

- den kurz- und langfristigen Folgen (z. B. erhoffte Stabilisierung und Regierbarkeit, danach aber möglicherweise Veränderung der politischen Strukturen). 
Diese Gegenstandsbereiche werden mit unterschiedlichen Systembeschreibungen modelliert. Die unterschiedlichen Beschreibungen der Teilsysteme sind formal meist nicht zu verketten, d.h. das Ergebnis der einen Systembeschreibung als Voraussetzung für die nachfolgende Systembeschreibung muss übersetzt werden. Diese Übersetzung geschieht überwiegend in Form des interdisziplinären Dialogs zwischen den Projektbeteiligten, gegebenenfalls mit weiteren Experten oder Betroffenen. Das bedeutet nicht, dass das Ergebnis dieser Diskussion unplausibel sein muss, aber die Diskussion ist in der Regel mit Konflikten verbunden, weil die mangelnde Formalisierbarkeit den Interpretationsspielraum erhöht und die wissenschaftliche wie politische Sozialisation der Beteiligten eine Rolle spielt (Berger und Luckmann 2009).

2. Man kann unter den Methoden der TA einen Mix von angemessenen wissenschaftlichen Verfahrensweisen verstehen. Dann erhält man bei einem Methodenmix, der sich aus den unterschiedlichen Systemtypen ergibt, nicht nur unterschiedliche Beschreibungssprachen, sondern auch unterschiedliche Beurteilungen der Angemessenheit der gewählten Methoden. Dies scheint der Kernpunkt der Auseinandersetzungen um die Validität der Ergebnisse der TA zu sein.

3. TA besteht nicht nur aus der wissenschaftlich fundierten Analyse der jeweils im Projektfokus stehenden Fragestellung, sondern ist auch gesellschaftliche Praxis, da sie Teil der Beratungspraxis in Politik, Wirtschaft und Gesellschaft geworden ist. Viele Analysen zum theoretischen Status untersuchen die Methoden der Beratungspraxis und verwenden dabei soziologische und politikwissenschaftliche Modellvorstellungen und Begrifflichkeiten. Andere Ansätze zur Analyse nehmen sich den Vorgang der Technisierung, d.h. die Durchdringung unserer Lebensvollzüge mit Technik vor, weil nur damit der Prozess, mit dem es die TA zu tun habe, verstanden werden könne. Dazu braucht man einen theoretischen Bezug zum technischen Wissen (Ropohl 2007). Der Konflikt entzündet sich daher an der Frage, ob der in einem Projekt tatsächlich vorliegende Methodenmix sich

\section{Auch in den Technikwissenschaften}

\section{ist angesichts der Digitalisierung eine Auflösung der methodischen Rigorosität hin zum Tentativen zu beobachten.}

an den Fragestellungen, den impliziten Erwartungen der Auftraggeber und an dem von den Beteiligten gefühlten oder vermeintlich antizipierten gesellschaftlichen Konsens bewusst oder unbewusst orientiert. Man weiß aus Studien zu Diskursen innerhalb von sogenannten Living-Labs, dass die wissenschaftliche und normative Sozialisation der am Projekt Beteiligten eine größere Rolle spielt als es der quasiobjektiv daherkommende Mitteilungsstil der Ergebnisberichte vermuten lässt (zur Laborarbeit allgemein Latour und Woolgar 1986; zu Living Labs speziell Kornwachs 2014).

Das nichttriviale Problem bei einem Methodenmix, wie er bei der Vorgehensweise der TA auftritt, liegt daher nicht in der bloßen Existenz unterschiedlicher Methoden, die nacheinander abgearbeitet werden könnten. Er liegt vielmehr darin, dass die in der TA bekannten Arbeitsschritte in der Projektpraxis iterativ und sich selbst modifizierend mit entsprechenden Kontrollund Korrekturschleifen durchlaufen werden müssen. Damit werden die Ergebnisse, die mit einer Methode aufgrund einer Systembeschreibung gewonnen worden sind, nun Ausgangspunkt für die Bearbeitung einer weiteren, anderen Systembeschreibung mit einer anderen Methode.

Dieses Problem ist in der interdisziplinären Zusammenarbeit, sei es der Forschung oder technologischen Entwicklungsarbeit, keinesfalls neu und hat schon früh zu Versuchen geführt, z. B. Systemtheorie als Brücke zwischen den Wissenschaften und Disziplinen zu sehen (Frank 1965). Aus dem Hype der Kybernetik, Futurologie und General System Theory der 1970er-Jahre blieb die wissenschaftstheoretisch inspirierte Grundlegung der Methodik der Modellbildung übrig (Klir und Elias 1985; Zeigler et al. 2000), die auch die Frage nach der Geeignetheit des gewählten Modelltyps für die Fragestellung systematisiert und nachvollziehbar zu beantworten versucht. In der Szenariotechnik sind in der Tat viele dieser systematischen Überlegungen zur Modellbildung berücksichtigt worden, wobei in der Praxis deren mathematische Durchdringung nicht immer geleistet werden kann: Nicht jedes Szenario führt zu einem Simulationsmodell.

\section{Modellbildung}

Wissenschaftstheoretisch gesehen kann das Standardvorgehen der TA im ersten Teil eines Projekts als iteratives Vorgehen von Systemanalyse und Systemsynthese angesehen werden. Es mündet in ein Modell, das mit n-fachen Alternativen bezüglich weniger Parameter variiert. Diese Varianten repräsentieren verschiedene mehr oder weniger erwartbare Zukünfte (acatech 2012; Grunwald 1999). Diese können sich sowohl auf die Technologieentwicklung als auch auf deren Folgen beziehen. Quantitative Verfahren zur Erfassung von Technologieständen sind schon früh entwickelt worden (Grupp et al. 1987).

Gerade im Bereich der Digitalisierung, in der sich aufgrund der fast grenzenlosen Möglichkeiten der Vernetzung und der oben erwähnten Ersetzung fast beliebige technische wie administrative Bereiche miteinander verknüpfen lassen, stoßen bei der Modellbildung verschiedene Wissensarten zusammen. Dabei vermischen sich wissenschaftliches Wissen (ausdrückbar als wissenschaftliche Erklärungen oder Gesetze $[\mathrm{A} \rightarrow \mathrm{B}]$ ), konstruktives Wissen (Verfahren nach Regeln B per A) und praktisches Wissen (how to do A). Eine Verknüpfung dieser Wissensarten im Bewusstsein eines Projektingenieurs oder eines Teams, das 
TA betreibt, ist in der Praxis fast problemlos der Fall, theoretisch wurde diese „Wissensintegration“ aber erst in den Anfängen untersucht (Meinold 2006). Zu diesem Wissensmix tritt auf der anderen Seite bei der Modellierung von technischen Entwicklungen und ihren Auswirkungen die Notwendigkeit hinzu, die Wechselwirkung von Systemen mit ökologischen, ökonomischen, sozialen oder technisch-organisatorischen Gegenstandsbereichen zu analysieren, von Systemen also, die auf unterschiedlichsten Modellbildungsebenen abgebildet werden müssen.

Auch in den Technikwissenschaften ist angesichts der Digitalisierung eine Auflösung der methodischen Rigorosität hin zum Tentativen zu beobachten. Allein die Herausforderung, viele bisher mechanisch, elektrisch oder gar elektronisch realisierbare technische Funktionen informationstechnisch oder salopp ausgedrückt, digital darzustellen und zu realisieren, erzwingt ein Neubedenken der bisherigen Funktionalitäten, was zum Teil zu völlig neuen Gerätedefinitionen und Geschäftsmodellen führt. Die Erkenntnis, dass man nicht gegen die Physik konstruieren kann, wird durch die Erkenntnis ergänzt, dass man aus der Physik (generell Naturwissenschaft) Technik und ihre Gestaltungsmöglichkeiten nicht ableiten kann. Hier haben die Technikwissenschaften den Aufweis der Machbarkeit vor die formale Beweisbarkeit gesetzt, und diese Priorisierung lässt sich auch durch wissenschaftstheoretisch logische Analysen der Strukturen technischen Wissens durchaus begründen.

Das bedeutet, dass sich ein Methodenmix und eine daran orientierte Modellbildung für die TA aus der Brauchbarkeit für die Problemstellung rechtfertigten. Dazu gehört, Wissen mit noch tolerierbaren Graden von Unbestimmtheiten $\mathrm{zu}$ verwenden, wobei auch Wissen eine Rolle spielt, das aus bloßen Abwägungen stammen kann. Es darf dabei allerdings nach wie vor gefordert werden, dass das Gesamtvorgehen nachvollziehbar, d.h. dann auch im Sinne von Karl Popper kritisierbar sein muss.

\section{Bewertungen}

In der zweiten Hälfte von TA-Projekten (gerade bei der prospektiven TA) werden Bewertungen der Folgen der n-fachen Alternativen aufgrund von gewählten normativen Vorgaben vorgenommen. Dies würde im Idealfall konsequentialistisch geschehen, d. h., man würde deduktiv Klassen von erwünschten und nicht erwünschten potenziellen Folge und Nebenfolgen bilden und dann durchdiskutieren.

Dies ist in der Regel jedoch nicht möglich, weil gerade hier der Übergang von der Methode des meist quantitativ bestimmbaren Modells (z. B. Simulation und deren Ergebnisse) zur induktiven Methode der Schätzung liegt. Dabei können durchaus Erhebungen, Umfragen, Experten-Delphi, szenische Darstellungen etc. weiterhelfen, man hat es aber in der Regel mit qualitativen Aussagen zu tun. Hier ist die Statistik weniger entscheidend, sondern welche Fragen aufgrund des obigen Modells und der Szenarien den Experten überhaupt gestellt werden können. Man braucht für die Konstitution des Gegenstandsbereichs der möglichen Folgen daher das, was man in der Wissenschaftstheorie eine Vortheorie nennt.

Den methodischen Kern der nachfolgenden Bewertung kann man ebenfalls in den Verfahren der Nutzwertanalyse (s. o.) finden. Entscheidend ist, dass man ein operatives Verfahren angibt (z.B. VDI 1991), das gegebenenfalls mit der gleichen Datenlage auch von anderen Arbeitsgruppen wiederholt werden kann. Das bedeutet, dass Technikbewertung eine Teilmenge des Arbeitsplans der TA darstellt.

Hier ist allerdings ein wesentlicher Unterschied $\mathrm{zu}$ beachten: Während die Wiederholung des Projektschritts der Modellbildung bei gleichen Prämissen zu einem vergleichbaren Modell führen müsste, können die Wiederholungen der Bewertung stark variieren, da hier unterschiedliche Wichtungen selbst bei gleichem Wertekanon eine entscheidende Rolle spielen. Replizierbarkeit und Reproduzierbarkeit fallen also nicht zwangsläufig zusammen. Deshalb ist es für den Rezipienten eines TA-Ergebnisses wichtig, den Wertekanon der Projektgruppe zu kennen.

\section{Ausblick}

Während die wissenschaftstheoretische Zuordnung der Methoden und der Arbeitsschritte zu den Technikwissenschaften samt einer Einbettung der herangezogenen Disziplinen im ersten Teil von TA-Projekten vergleichsweise einfach vorgenommen werden kann, ist die Vorgehensweise der Bewertung im zweiten Teil in der Praxis oftmals undurchsichtig.

Dabei muss gesagt werden, dass die Praxis der TA, gerade da, wo sie den gegenseitigen Einfluss von Technikentwicklung und gesellschaftlichen Prozessen untersucht, zwar im methodischen Kern wissenschaftlich vorgeht, also eine technikwissenschaftliche Disziplin sein kann, aber als Praxis allein noch keine Wissenschaft, also auch keine Wissenschaft von der Technik ist - Praxis setzt hier Wissenschaftlichkeit voraus. TA trägt jedoch bei aller Ungewissheit zur Selbstvergewisserung der Technikwissenschaften bei, indem sie zu einer Aufklärung über die Wechselwirkung von Technik und organisatorischen Wechselwirkungen (d. h. ihrer organisatorischen Hülle) und damit über die Bedingung der Möglichkeit technischer Gestaltung beiträgt.

Sicher wäre zur Vervollständigung der wissenschaftstheoretischen Durchdringung eine vereinheitlichende Vorgehensweise wünschenswert, die normative Vorgaben, z. B. wie in der Technikbewertung (VDI 1991), der Soziologie (Kornwachs und Renn 2011), der Ethik (Betz und Cacean 2011) etc. mit operational definierbaren Kriterien und diese mit Indikatoren verknüpft.

Bei der Erfüllung solcher methodischer Standards, die Kritisierbarkeit, Transparenz und Nachvollziehbarkeit - im Idealfall sogar ein ähnliches oder zumindest vergleichbares Ergebnis bei der wiederholten Projektdurchführung - ermöglichen sollen, kann das Vorgehen der Technikfolgenabschätzung auch als eine Teildisziplin der Technikwissenschaften angesehen werden. Das würde ihre Integration in das Curriculum der Technikwissenschaften erleichtern. 


\section{Literatur}

acatech - Deutsche Akademie der Technikwissenschaften (Hg.) (2012): Technikzukünfte. Vorausdenken - Erstellen - Bewerten. acatech IMPULS, November 2012. Online verfügbar unter: http://www.acatech.de/fileadmin/user_ upload/Baumstruktur_nach_Website/Acatech/root/de/Publikationen/ Stellungnahmen/P_2012-11_acatech_Technikzukuenfte_WEB_25102012.pdf, zuletzt geprüft am 14.02.2018.

acatech - Deutsche Akademie der Technikwissenschaften (Hg.) (2013): Technikwissenschaften. Erkennen - Gestalten - Verantworten. acatech IMPULS, November 2012. Online verfügbar unter: http://www.acatech.de/fileadmin/ user_upload/Baumstruktur_nach_Website/Acatech/root/de/Publikationen/ Stellungnahmen/acatech_IMPULS_Technikwissenschaften_WEB_final.pdf, zuletzt geprüft am 14.02.2018.

Banse, Gerhard; Grunwald, Armin; König, Wolfgang; Ropohl, Günter (Hg.) (2006): Erkennen und Gestalten. Eine Theorie der Technikwissenschaften. Berlin: edition sigma.

Berger, Peter L.; Luckmann, Thomas (2009): Die gesellschaftliche Konstruktion der Wirklichkeit. Eine Theorie der Wissenssoziologie. Frankfurt a. M.: S. Fischer.

Betz, Gregor; Cacean, Sebastian (2011): The moral controversy about Climate Engineering. An argument map. Karlsruhe: KIT. Online verfügbar unter: http://digbib.ubka.uni-karlsruhe.de/volltexte/1000022371, zuletzt geprüft am 09. 02.2018.

Bunge, Mario (1967): Scientific research II. The search for truth. Berlin: Springer. Decker, Michael (2007): Praxis und Theorie der Technikfolgenabschätzung. Erste Überlegungen zu einer methodischen Rekonstruktion. In: TATuP - Zeitschrift für Technikfolgenabschätzung in Theorie und Praxis 16 (1), S. 25-34.

Frank, Helmar (Hg.) (1965): Kybernetik - Brücke zwischen den Wissenschaften. Darmstadt: Wissenschaftliche Buchgesellschaft.

Gethmann, Carl F.; Grunwald, Armin (1996): Technikfolgenabschätzung. Konzeptionen im Überblick. Bad Neuenahr-Ahrweiler: Europäische Akademie (Graue Reihe, 1).

Grunwald, Armin (Hg.) (1999): Rationale Technikfolgenabschätzung. Berlin u. a.: Springer.

Grunwald, Armin (2007 a): Auf dem Weg zu einer Theorie der Technikfolgenabschätzung: der Einstieg. Einführung in den Schwerpunkt. In: TATuP - Zeitschrift für Technikfolgenabschätzung in Theorie und Praxis 16 (1), S. 4-17.

Grunwald, Armin (2007 b): Die Funktion der Wissenschaftstheorie in der Technikfolgenabschätzung. Karlsruhe: ITAS Pre-Print. Online verfügbar unter: www. itas.kit.edu/pub/v/2007/epp/grun07-pre03.pdf, zuletzt geprüft am 09.02.2018.

Grunwald, Armin (2007 c): Die konstitutive Rolle von Technik in der konstruktivistischen Wissenschaftstheorie. Konsequenzen für die Technikphilosophie. In: Journal for General Philosophy of Science 38, S.239-259. DOI: 10.1007/s10838006-9025-2.

Grupp, Hariolf; Hohmeyer, Olaf; Kollert, Roland; Legler, Harald (1987): Technometrie. Die Bemessung des technisch-wirtschaftlichen Leistungsstandes. Köln: TÜV Rheinland.

Janich, Paul (1997): Kleine Philosophie der Naturwissenschaften. München: C. H. Beck.

Klir, George; Elias, Doug (1985): Architecture of systems problem solving. New York: Plenum Press.

Kornwachs, Klaus (2006): Rational-systematische Methoden. In: Gerhard Banse, Armin Grunwald, Wolfgang König und Günter Rohpohl (Hg.): Erkennen und Gestalten. Eine Theorie der Technikwissenschaften. Berlin: edition sigma, S. 145-159.
Kornwachs, Klaus (2012): Strukturen technologischen Wissens. Analytische Studien zu einer Wissenschaftstheorie der Technik. Berlin: edition sigma.

Kornwachs, Klaus (2014): Innovationsprozesse in Living Labs: Theorie und Empirischer Zugang. Bericht II zum Projekt ST-BMDD: „Systemische Technikgestaltung: Begriffe - Methoden - Definitionen - Design“ an das FraunhoferInstitut für Arbeitswirtschaft und Organisation, Stuttgart. Büro für Kultur und Technik. Argenbühl, Februar 2014

Kornwachs, Klaus; Renn, Ortwin et al. (2011): Akzeptanz von Technik und Infrastrukturen. Anmerkungen zu einem aktuellen gesellschaftlichen Problem. acatech - Deutsche Akademie der Technikwissenschaften, acatech bezieht Position - Nr. 9., Berlin. Online verfügbar unter: http://www.acatech.de/ fileadmin/user_upload/Baumstruktur_nach_Website/Acatech/root/de/ Publikationen/Stellungnahmen/acatech_bezieht_Position_Nr9_Akzeptanzvon-Technik_WEB.pdf, zuletzt geprüft am 09.02.2018.

Kroes, Peter; Meijers, Anthonie (Hg.) (2000): The empirical turn in the philosophy of technology. Amsterdam: Elsevier.

Latour, Bruno; Woolgar, Steve (1986): Laboratory life. The construction of scientific facts. Princeton NJ: Princeton University Press.

Meinold, Natalja (2006): Wissensintegration und Handeln in Gruppen. Wiesbaden: Deutscher Universitätsverlag.

Mesarovic, Mihajilo D. (1972): A mathematical theory of general systems. In: George Klir (Hg.): Trends in general system theory. New York: Wiley-Interscience, S. 251-269.

Paschen, Herbert; Gresser, Klaus; Conrad, Felix (1978): Technology Assessment. Technikfolgenabschätzung. Frankfurt a. M.: Campus.

Poser, Hans (2017): Homo Creator. Technik als philosophische Herausforderung. Wiesbaden: Springer vs.

Ropohl, Günter (1999): Allgemeine Technologie. Eine Systemtheorie der Technik. München: Hanser.

Ropohl, Günter (2007): Theorie der Technisierung. In: TATuP - Technikfolgenabschätzung in Theorie und Praxis 16 (3), S. 115-119.

Shrader-Frechette, Karin. S. (1980): Technology assessment as applied philosophy of science. In: Science, Technology and Human Values (5), S. 33-50.

Tetens, Holm (1982): Was ist ein Naturgesetz? In: Allgemeine Zeitschrift für Wissenschaftstheorie 13 (1), S. 70-83.

VDI - Verein Deutscher Ingenieure (1991): VDI Richtlinie 3780 Technikbewertung. Begriffe und Grundlagen. Düsseldorf: VDI-Verlag.

Wright, Georg H. von (1994): Normen, Werte, Handlungen. Frankfurt a. M.: Suhrkamp.

Zeigler, Bernard P.; Praehofer, Herbert; Kim, Tag Gon (2007): Theory of modelling and simulation. San Diego: Academic Press.

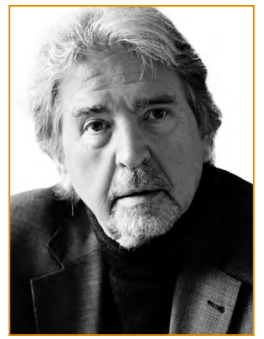

war bis 2011 Lehrstuhlinhaber für Technikphilosophie an der BTU Cottbus. Er forscht und publiziert auf dem Gebiet der analytischen Wissenschaftstheorie, der Ethik und der Technikentwicklung. Er lehrt derzeit an der Universität UIm. 